\title{
EMBEDDED FUZZY SYSTEMS IN WELDING POWER SOURCES
}

\author{
Marjan Golob ${ }^{1}$, Arpad Koves ${ }^{2}$, Boris Tovornik ${ }^{1}$ \\ ${ }^{1}$ University of Maribor, FERI, Smetanova 17, 2000 Maribor, Slovenia \\ ${ }^{2}$ Welding Institute, Ptujska 19, 1000 Ljubljana, Slovenia \\ E-mail: mgolob@uni-mb.si
}

\begin{abstract}
The paper discuss the possibilities of application of the fuzzy logic based embedded systems in welding power sources. The main characteristics of gas metal arc welding process are presented and the fuzzy reasoning based hierarchical decision support system for setting indirect welding parameters are described. Furthermore, the fuzzy logic based system for analysis of weld quality, particularly assessment of the weld surface condition by means of measurable electric signals acquired during welding are introduced. A simple fuzzy inference system was realized which could relatively efficiently assess the weld quality on the basis of time variations of the welding voltage, current and short-circuit time in a certain time window. Copyright (C) 2005 IFAC
\end{abstract}

Keywords: fuzzy system, decision support system, quality control, embedded systems, intelligent machines.

\section{INTRODUCTION}

The requirements for welding equipment are increasingly in favour of the welding systems permitting cost-cutting and high-quality production. The features of the welding device mainly depend on the device control unit and its software. A comparison of the latest welding devices and of the conventional ones will show major differences in the control processes applied. The features of the up-todate welding machines are software based parameter setting and on-line parameter adaptation during their operation. Because of the complexity of the welding process the programs often contain elements of information processing based on soft computing methods.

In (Yamamoto et al., 1996) a $\mathrm{CO}_{2} / \mathrm{MAG}$ welding device with a controller based on fuzzy inference which provides better welding results than the conventional devices was described. The method is based on the application of a multi-characteristic control reducing material spatter. It consists of two control circuits, one for fuzzy control of the welding voltage and the other for that of the welding current providing the process stability due to the variation of the penetration depth by change of an electrode extension. In (Mita et al., 1996) authors described a method of automatic setting, i.e. adaptation, of the welding voltage of the welding machine in order to maintain optimum welding conditions during the welding process. The decision system is based on the fuzzy logic system and describes the relations among the welding-process condition, the welding voltage set, the standard deviation of short-circuit time, and the standard deviation of the arc-burning time. In (Zhang and Wang, 2001) authors treated an inverter welding machine for $\mathrm{CO} 2$ welding with a flat characteristic and independent arc-stability control, bead-formation control, and material-spatter control. The stabilisation of the arc length is provided by the algorithm of the control of an electric welding signal pattern based on the fuzzy logic methods.

On-line monitoring of weld quality in the arc welding process is next very interesting industrial application since it permits to cut the production costs and the risk of low-quality welded structures. The basic task of the system is to analyse the weld quality or to simultaneously classify the welds concerned, on the 
basis of measurable information, in those free of defects and those showing defects. The precision of the classification depends on the amount and quality of the information acquired in the welding process going on. The larger is the amount of information in the welding process, the more precise is the classification of the welds. In the market there are several commercial systems available permitting online quality monitoring. It is common for the majority of them that they simultaneously measure welding voltage, welding current, and some other process quantities, and then compare them to the preset nominal values. In case of a too strong deviation of a quantity from the preset nominal value, the quality analyser will signalize a defect and transmit the signal to the system control unit. The investigation follows from the assumption that the entire information in the welding process can be supplied by the time variations of the welding voltage and current. A signal analysis provides characteristic information on the quality of the welding process by providing the measured variations of voltage and current. The main methods of signal analysis are the following:

- statistical methods in time and frequency domains (mean, standard deviation, various distributions and signal histograms etc.)

- signal frequency analysis (Fourier transform, some other transformations), in which various signal spectra and their harmonic components are processed more in detail etc.

- correlation analysis (autocorrelations, crosscorrelations of signals).

Computer-aided systems for welding-process monitoring can be generally applied:

- to monitoring of the welding process, i.e., manufacture (quality assurance),

- to support the development of filler materials, shielding gases and welding devices,

- to optimization of a welding process technology,

- to setting of parameters and characteristics of welding devices,

- to the adaptive control of the welding process and development of process sensors.

A universal application of the above-mentioned methods and systems is impossible. The today existing methods and systems are suitable only for the application to concrete cases, e.g. a concrete welding process, material transfer through the arc, work-piece type and parameters, type of filler material, shielding gas and similar. The method of measurement of the electric quantities and their comparison with the nominal values, however, is very often not enough to efficiently decide on the weld quality in arc welding. In paper (Rehfeldt et al., 2000; Wu et al., 2001) authors suggested statistical processing of the measured electric signals and the fuzzy decision inference system for the GMAW process quality on the basis of statistical indicators such as probability density distribution of the welding voltage and current, and class frequency distributions of the short-circuit times. The decision on the GMAW quality based on the statistical indicators requires complex knowledge of the welding process and the physics of the welding arc. Consequently, the authors proposed to realise the decision inference system in two ways, i.e., the fuzzy inference system and the Kohonen neural network. The experimental results obtained in testing of the two methods showed $92 \%$ efficiency in the identification of defects in the GMAW process.

\section{GMAW PROCESS}

In most cases arc welding is related to a process which can be performed through GMAW. A fundamental significance of GMAW process is that it incorporates automatic feeding of a consumable electrode that is protected by an externally supplied shielding gas as is presented in Figure 1.

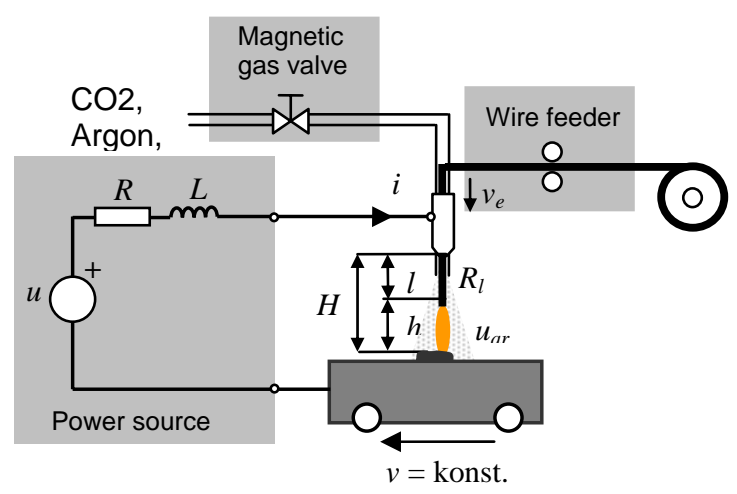

Fig. 1. Principle of GMAE process

During operation the GMAW process must be regulated with respect to arc length $h$ and wire feed speed $v_{e}$ to obtain stable conditions during welding. GMAW process have basically three means by which metal is transferred from electrode to the work, namely: short circuit transfer, globular transfer, and spray transfer. The type of transfer mode is affected by a number of parameters in the welding process of which magnitude ant shape of welding current, electrode diameter, electrode composition, electrode extension (contact-tip-to-work-distance CTWD) and shielding gas are the most important.

Short circuit transfer mode is mostly used for thin segments and overcoming large joint gaps. It is affected by lowest range of welding currents and electrode diameters. During short circuit transfer, the welding current increases when the wire contacts the weld metal. The current raise must be controlled to avoid spatter during short circuit which otherwise shows an explosion type of behaviour. The open circuit voltage of the power source must be low enough so that the drop of molten metal of the wire can not transfer until it touches the base metal. A number of short circuits are in general in the order of a few to a few hundreds per second. 
Globular transfer mode is characterised by a drop size with a diameter greater than the electrode. The average current is slightly higher than for short circuit and the arc should be long enough to ensure detachment of the drop before it contacts the weld pool. The number of drops is low - a few per second. The main characteristics of globular transfer mode are lack of fusion and inefficient penetration. It has a little use in practical applications.

Spray transfer is characterised as a spatter-free spray. The current should be DC with positive electrode and with magnitude high enough to assure transfer consisting of very small drops. The transfer rate is several hundreds drops per second. Stabile axial spray is possible to obtain in shielding gas which is reach with argon. A limitation of the spray transfer is relatively high penetration which makes it improper to weld thin plates.

In paper (Cook et al., 1992) authors separates the variables of the welding process into two categories: direct weld parameters (DWP) and indirect weld parameters (IWP). The DWP are those pertaining to the weld reinforcement and fusion zone geometry, mechanical properties of the completed weld, weld microstructure, and discontinuities. The weld pool width, penetration depth, porosity, ductility, etc. are member of the DWP. The indirect weld parameters are those input variables that collectively control the direct weld parameters, e.g., voltage $u$, current $i$, welding travel speed $v$, electrode feed rate $v_{e}$, and electrode extension $l$, etc. The disturbance parameters can be varied on line during welding to affect change in DWP so they must be controlled by the IWP. Since a nonlinear relationship is characteristic for connection between DWP and IWP, the coefficients of the relation models that relate DWP and IWP vary as a function of operating conditions and disturbances. Hence, the feedback control systems with capability of adaptive parameter adjustment and supported with easy and intelligent user interface for setting IWP are necessary to maintain stable control over the operating range of the GMAW process.

There have been many attempts to model the GMAW process. For on-line control of the GMAW process an accurate dynamic model that provides guidance for sensing and corrective action is needed. The mathematical model developed in (Golob et al., 2002) consists of four components: GMAW current model, burn-off model, droplet transfer model and weld pool width model. The model is applicable to testing different control algorithms and intelligent methods for setting IWP.

\section{FUZZY CONTROL OF GMAW PROCESS}

Within most welding production today, the designer of the welded part specifies the desired weld characteristics (setting the DWP). The job of the welding engineer then is to determine a set of indirect weld parameters. There are two aspects of setting IWP: ability to specify directly to the welding system computer the desired DWP, and implementation of feedback control of the important DWP. Successful implementation of multivariable weld control involves sensing, modelling, and control. Practical weld process control implementation involves a substantial amount of condition or heuristic logic, particularly with multivariable and adaptive control.

The sense of the soft computing control (fuzzy systems, neural networks, genetic algorithms, etc.) concepts is to provide a systemic approach to dealing with many conditions that must be built into most practical welding control systems. In recent years the welding power source technology has been progressed particularly remarkable and most of power sources has been converted to digital control enabling the connection with robot and other external control units ant various IT devices. The IWP settings can be realised in a hierarchy as shown in Fig 2.

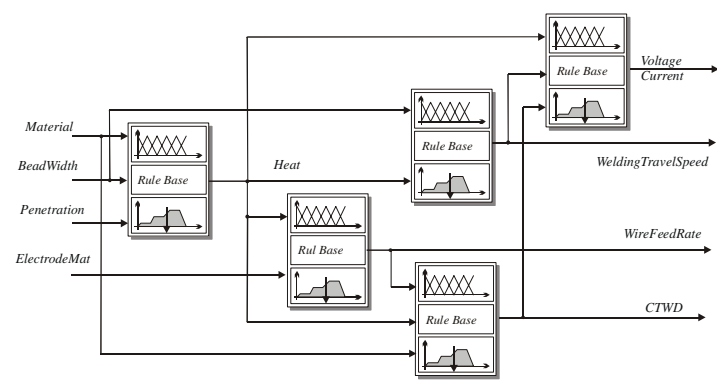

Fig.2. The fuzzy reasoning based hierarchical decision support system.

In short the system works as follows. Given the current state of the DWP, the fuzzy decision support system generates states of the IWP and presents them to the welder who should accept them or partly change them. The role of the fuzzy decision support system is to suggest welder initial states of IWP.

\section{FUZZY LOGIC BASED SYSTEM FOR QUALITY MONITORING}

Fundamental tasks of the system for the analysis of the welding-process condition are automatic monitoring and recognition of characteristic parameters of the welding process and determination of the weld quality or on-line classification of welds into defective or non-defective groups. The accuracy of weld classification depends on the quantity and quality of information available in the welding process. The more comprehensive the information in the process is, the more accurate the weld classification can be. Consequently, a large variety of sensors based on electric, optical and acoustic measurements are applied. The use of the majority of 
such sensors, however, is expensive and the applicability of the sensors is limited to laboratory conditions. In our research it was assumed that the complete information in the welding process is recorded by the time variations of welding voltage and current. The on-line measurement of the welding voltage and current is relatively simple and feasible. The characteristic information in the welding process can be obtained by an analysis of the measured variations of the welding voltage and current signals. Fig. 3 shows a block schematic diagram of the analyzer.

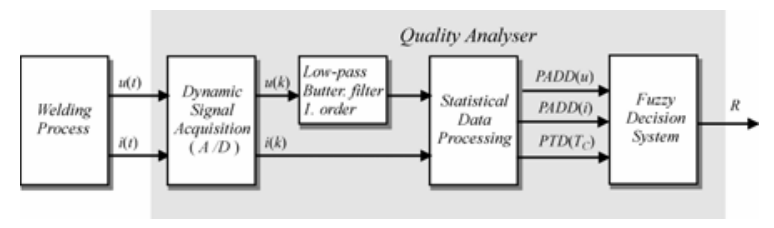

Fig. 3. Block diagram of the system for automatic classification of characteristic welding parameters.

The time variations of the measured signals, i.e., in our case the welding voltage and current, are not suitable for direct processing by a fuzzy system. The signals have to be first transformed into information more suitable for the fuzzy system.

As a characteristic piece of information on the time variations of the welding voltage and current, the probability amplitude-density distribution (PADD) was selected. The simplest way to assess the function of the PADD for a signal is to plot a histogram of the particular signal representing a final number of intervals between the minimum amplitude and the maximum one. The purpose of the PADD functions is to show clearly some characteristics of the signal variations in the time domain. A piece of information important to determination of the weld quality is the change in short-circuit time $T_{C}$. In a similar way as in the analysis of the probability amplitude distribution, the density time distribution (PTD) of short-circuit times $T_{C}$ was defined within the time window.

An analysis of PADD functions of the welding voltage and current and an analysis of the distribution of short-circuit times showed that a disturbance in the welding process was expressed in different zones of the PADD functions. Four PADD zones of the welding voltage, two PADD zones of the welding current and two zones of the distribution of short-circuit times $T_{C}$ were analyzed. Characteristic variables were introduced for the individual zones which were defined as a sum of PADD values of the individual intervals within the zone. These characteristic variables were used to analyze the welding process and infer the weld quality. The system for the analysis of the welding process in terms of the weld quality is based on comprehensive expert knowledge which should be recorded in a way suitable for computer processing.
4.1 Determination of the weld quality by means of the fuzzy inference system

Any fuzzy system consists of the following elements: fuzzifying of the input variables, fuzzy inference based on fuzzy rules, and defuzzifying of the output variables. Fuzzifying of input variables is a map of the crisp input signals to the fuzzy linguistic quantities. The definition of the linguistic quantities is important to understanding of fuzzy rules and fuzzy inference. At the same time it permits a "verbal" description of the problem. This is important in the implementation of expert knowledge in the operation of the fuzzy system. Fuzzy inference is a process of inference in which the fuzzy input quantities are mapped in accordance with the fuzzy rules, collected in a fuzzy rule base, into the fuzzy output quantities. Defuzzification is a process mapping the fuzzy output quantities (result of inference) into the defuzzified quantities.

In the case of three linguistic input variables, $I N_{1}$, $I N_{2}$, and $I N_{3}$, one output variable, $O U T$, and when five fuzzy sets [very_small, small, medium, large, very_large] are defined on each definition space of linguistic variables then only $5^{3}=125$ of rules can be determined in the rule base with following rules:

if $I N_{1}=v e r y \_s m a l l$ and $I N_{2}=v e r y \_l a r g e$ and $I N_{3}=$ very_large then OUT = very_large.

In our fuzzy inference system the Mamdani type of inference was realised with the min accumulation operator, the max aggregation operator, the min implication function, the COG (center of gravity) deffuzification method, and triangular membership functions of the fundamental fuzzy sets.

\subsection{Experiment results}

In order to analyze the gas-shielded arc welding process, a power source VPS 5000 (Var Puls Synergic) was used. Welding was carried out using the short-circuit GMAW process on a $10 \mathrm{~mm}$ thick St 52 material (size of workpieces: $200 \mathrm{~mm}$ x $500 \mathrm{~mm}$ ), a wire filler material, VAC 60, of $1.2 \mathrm{~mm}$ in diameter, and a two-component gas mixture, 92\% $\mathrm{Ar}$ and $8 \% \mathrm{CO}_{2}$. The main part of the experiment was consisted of surfacing on a flat plate and simultaneous acquisition of data. The first half of the surfacing weld was made on a suitably prepared, i.e., sand blasted, plate whereas its second half was made with the same welding parameters on a plate covered with scales, which represented a disturbance. The technological evaluation of the welding cases consisted of a visual inspection and non-destructive testing of the surfacing welds made. This means that the surfacing welds made were first assessed visually in terms of the surface appearance and then subjected to an X-ray examination. Inspection results are divided into two groups: sandblasted substrate (nice surface appearance, radiograph - no particularities n.p.) and substrate covered by scale (rough surface of 
the surfacing weld and occurrence of strong side spatters, radiograph - no particularities - n.p. as is shown on Fig. 4. The following assessment is made: although the radiographs of the surfacing welds showed no particular imperfections, the weld quality on the scaled surface was poor. In all cases a rough surface and the occurrence of strong spatters can be observed.

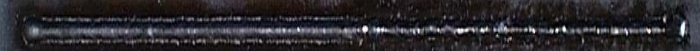

Fig. 4. Photo of the surfacing weld.

The electrical signals were captured by a 2-channel $60 \mathrm{MHz}$ digital oscilloscope. The voltage was via an adequate filter supplied to the oscilloscope and the welding current via a Hall probe to the oscilloscope input. The sampling frequency for each signal was equal to $100 \mathrm{kHz}$. After being captured the signals were stored on a storage medium to be later used in data processing. The analysis of welding signals included the use of software tools, LabVIEW and Matlab - Simulink, for which corresponding software applications for signal processing was elaborated. Each experiment was recorded in a file consisting of three respective columns showing data on time, voltage and current signals. The table contained 1.000.000 rows, which indicates an experiment time of $10 \mathrm{~s}$ and a sampling frequency of $100 \mathrm{kHz}$. The time variations of the signals were analyzed in a time window of $200 \mathrm{~ms}$. That means that the time variations are monitored for $200 \mathrm{~ms}$, then a statistical data analysis is made, and then with the fuzzy inference system the weld quality is inferred.

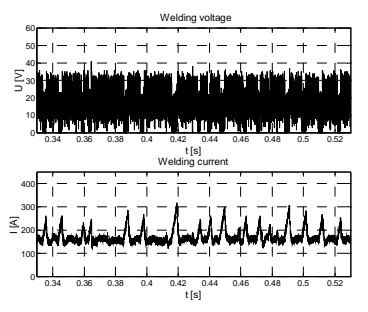

a.

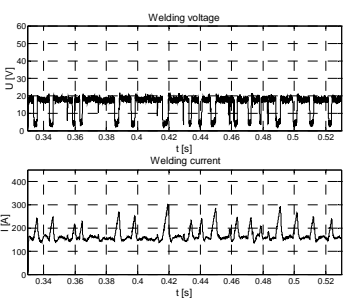

b.
Fig. 5. Measured voltage and current in time window from $0.33 \mathrm{~ms}$ to $0.53 \mathrm{~ms}$. a) before filtering; b) after filtering with Butterworth filter of $1^{\text {st }}$ order and limit frequency $f_{C}=1000 \mathrm{~Hz}$.

Fig. 5a shows voltage and current within the time window of $200 \mathrm{~ms}$. The frequency of the chopper, being strongly expressed in the oscillograms of the welding voltage and current, was eliminated by a Butterworth digital filter. Thus more clear oscillograms in the time domain were obtained (Fig. 5b). With lower welding-current settings, the occurrence of short circuits was clearly visible. When a short circuit occurs, the arc will extinguish, the welding voltage drops strongly whereas the welding current will increase as long as the arc will ignite again. With the set parameters the material transfer will occur during the short circuit. The time variations of the signals do not permit a direct inference on the weld quality. An adequate statistical processing of the measured signals should be made. As a characteristic piece of information on the time variations of voltage and current, the probability amplitude-density distribution (PADD) was selected. The easiest way of obtaining the assessment of a PADD function is to plot a signal histogram in terms of its amplitude with a final number of intervals between the minimum amplitude and the maximum one. An example of the histogram of the time variation of voltage is shown in Fig. 6a. A change in amplitude from $0 \mathrm{~V}$ to $60 \mathrm{~V}$ was assumed. 121 intervals were selected within the range. The histogram shows that the voltage within the time window was varying from $0 \mathrm{~V}$ to $25 \mathrm{~V}$. The amplitude density was greatest around $18 \mathrm{~V}$ and with the short-circuit voltage, i.e., around $4 \mathrm{~V}$. A similar analysis was made with the measured current. The histogram of the current-amplitude distribution within the time window of $200 \mathrm{~ms}$ is shown in Fig. $6 \mathrm{~b}$. The next piece of information being of great use in the determination of the process condition, i.e., the weld quality during welding, is a change of the shortcircuit time $T_{C}$. The welding voltage oscillogram in Fig. 5 shows that $T_{C}$ varied in the time window of 200 ms from very short times to times of a few milliseconds. Similar to the analysis of the probability amplitude density distribution, an analysis of the probability distribution of the shortcircuit times $T_{C}$ within the time window was made. On Fig. 6c a histogram of $T_{C}$ distribution, which was calculated by dividing the range from 0 to $10 \mathrm{~ms}$ into forty classes is shown.

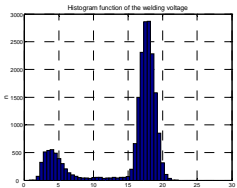

a.

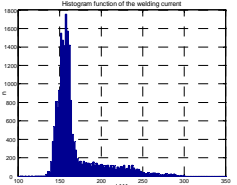

b.

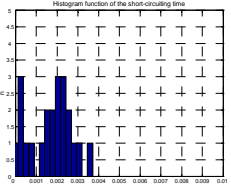

C.
Fig. 6. Histograms of welding signals: a) voltage, b) current, c) distribution of short-circuit times.

An analysis of the PADD functions of the welding voltage and current and an analysis of the distribution of short-circuit times showed that a disturbance in the welding process was expressed in different zones of the PADD functions. Figures 7a, 7b, and 7c show

PADD function of the welding voltage, PADD function of the welding current, and the distribution of the short-circuit times respectively. The signals were recorded with two characteristic welding processes, i.e., the one giving an acceptable weld and the other giving an unacceptable weld). In order to facilitate the comparison of the amplitude-density distribution of different measurements, the PADD functions were calculated, expressed in percent, and plotted in the logarithmic scale. The dotted curves represent the signals measured during an undisturbed welding process, which resulted in a good weld. The solid curves represent the signals measured during a 
disturbed welding process, which resulted in an unacceptable weld.

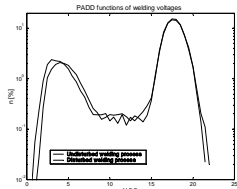

a.

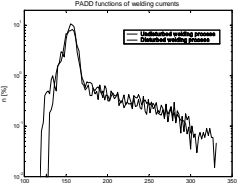

b.

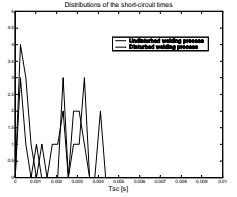

C.
Fig. 7. PADD functions of welding signals: a) voltage, b) current, c) distribution of shortcircuit times.

The figures clearly show the zones in which the functions differ. This difference was made use of to infer the weld quality. Four PADD zones of the welding voltage, two PADD zones of the welding current and two zones of the distribution of shortcircuit times TC were analyzed. Characteristic variables were introduced for the individual zones which were defined as a sum of PADD values of the individual intervals within a zone. Thus eight variables were obtained. They are given in Tab. 1.

\begin{tabular}{ll} 
Table 1 & Characteristic variables of the individual \\
& \multicolumn{2}{c}{ zones of PADD functions. } \\
\hline$V U 1$ & $\sum$ PADD of voltage from $0 \mathrm{~V}-4.5 \mathrm{~V}$ \\
$V U 2$ & $\sum$ PADD of voltage from $4.5 \mathrm{~V}-10 \mathrm{~V}$ \\
$V U 3$ & $\sum$ PADD of voltage from $10 \mathrm{~V}-18 \mathrm{~V}$ \\
$V U 4$ & $\sum$ PADD of voltage from $18 \mathrm{~V}-25 \mathrm{~V}$ \\
$V I 1$ & $\sum$ PADD of current from $100 \mathrm{~A}-150 \mathrm{~A}$ \\
$V I 2$ & $\sum$ PADD of current from $250 \mathrm{~A}-350 \mathrm{~A}$ \\
$V T 1$ & $\sum$ PADD of time $T_{C}$ from $0 \mathrm{~ms}-2 \mathrm{~ms}$ \\
$V T 2$ & $\sum$ PADD of time $T_{C}$ from $2 \mathrm{~ms}-10 \mathrm{~ms}$ \\
\hline
\end{tabular}

A circumstantial analysis (Koves and Golob, 2002) of the PADD functions indicates that the characteristic quantity VU2 is quite suitable for the determination of weld quality, VU1 and VT2 are suitable whereas the values of the other characteristic quantities unexpressively differ depending on the weld quality. Consequently, a decision was taken to elaborate the fuzzy system based on characteristic quantities VU1, VU2, and VT2. In the research thus the simple fuzzy decision system based on Mamdani type of fuzzy inference was realized with three fuzzy input variables and one fuzzy output variable (Fig. $3)$. The applicability of the fuzzy decision system was tested with the same set of the measuring data. The test result indicates that the inference system assessed the weld quality from the measurement signals correctly in approximately $80 \%$ of the cases.

\section{CONCLUSION}

Possibilities of application of artificial-intelligence methods in the field of modelling of technological processing such as welding are inexhaustible. In future they will certainly represent an important step ahead in the development of robotic welding systems. The present research aimed at indicating potential applications of the fuzzy logic to the analysis of the weld quality by means of the measurable electric signals. Thus a simple fuzzy system was elaborated, which was able to quite efficiently assess the weld quality on the basis of the time variations of the welding voltage and current and the short-circuit times in a particular time window. The system included 125 rules, which were chosen by experience and without an additional optimization. Better results would undoubtedly be obtained by taking into account the expert knowledge. A next possibility to improve the system is the introduction of algorithms of automatic system learning or automatic rule generation in the rule base.

\section{REFERENCES}

Cook G. E., Andersen K. and Barrett R. J. (1992). Keynote Address Feedback and Adaptive Control in Welding. International Trends in Welding Science and Technology, ASM International, pp. 891-903.

Golob M., Koves A., Puklavec A. and Tovornik B. (2002). Modelling, simulation and fuzzy control of the GMAW welding process. Preprints of the 15th IFAC World Congress, Barcelona, Spain, July 21-26.

Koveš A., Golob M. (2002). Fuzzy Logic Based Quality Monitoring in Short-Circuit Gas Metal Arc Welding. IIW-Doc. XII -1712-02.

Mita T., Tanoue H., Kashima T. and Shinada T. (1996). Automatic Setting of Suitable Arc Voltage using Fuzzy Control, Automation Technology of Arc Welding, Welding Guide Book III, Technical Commission on Welding Processes, Japan Welding Society, II, pp. 121126.

Rehfeldt D., Polte T., Wu C. S. (2000). Welding Quality Determination by Using Fuzzy Logic or Neural Networks, IIW-Doc. XII-1651-00.

Wu C. S., Polte T., Rehfeldt D. (2001). A Fuzzy Logic System for Processing Monitoring and Quality Evaluation in GMAW, Welding Journal, 81(2): pp.:S33-S38.

Yamamoto H., Nitta A., Nakamata T. and Uezono T. (1996). Automatic Setting of Welding Conditions by Fuzzy Controlled CO2/MAG Welding Machine, Automation Technology of Arc Welding, Welding Guide Book III, Technical Commission on Welding Processes, Japan Welding Society, II, pp. 127-132.

Zhang J., Wang H. (2001). A novel welding inverter power source system with constant current output characteristic based on fuzzy logic control, Proceedings of the Fifth International Conference on Electrical Machines and Systems, ICEMS 2001, vol.1, pp.: 567 - 570. 